Flash : Le gène DMD (de la myopathie de Duchenne) code pour une protéine (la dystrophine) qui pourrait être associée à la fois, au niveau de la triade, à la membrane plasmique et aux citernes du réticulum endoplasmique. Louis Kunkel et al. [1, 2] font l'hypothèse que la dystrophine pourrait participer au couplage entre la perception du signal de dépolarisation membranaire et la libération de $\mathrm{Ca}^{++}$par lo réticulum endoplasmique. La maladie pourrait étre due alors à une perturbation de l'homéostasie du calcium. [1. Hoffman EP, et al. Cell 1987; 51 : 919-28.] [2. Hoffman EP, et al. Nature 1987 ; 330 : 754-9.]

\title{
Mécanisme et traitement de l'oxalose
}

L'hyperoxalurie primitive de type I est une maladie caractérisée par une erreur innée du métabolisme de l'oxalate, transmise selon le mode récessif autosomique. La production d'oxalate est accrue ; le taux sanguin est élevé ; l'excrétion urinaire d'oxalate est multipliée par 5 ou 10 . Le désordre métabolique entraîne une lithiase urinaire oxalocalcique, le dépôt d'oxalate de calcium dans les reins et leur destruction progressive, souvent dans l'enfance, plus rarement à l'âge adulte. Le dépôt d'oxalate de calcium (ou oxalose) s'étend à d'autres tissus, comme l'appareil cardiovasculaire et l'os.

Pendant longtemps, on a considéré à tort que le déficit enzymatique responsable touchait la 2-oxoglutarate : glyoxylate carboligase cytosolique. Récemment, Danpure et al. [1, 2] ont corrigé cette " erreur " et ont montré que le déficit 'portait en fait sur l'alanine : glyoxylate aminotransférase, enzyme présente normalement dans les peroxysomes hépatiques. Les formes les moins sévères de la maladie s'accompagnent d'une certaine activité enzymatique résiduelle alors que ce déficit est plus complet dans les formes les plus graves et précoces. L'hyperoxalurie primitive de type I (résistant à l'administration de pyridoxine ou vitamine B6) prend ainsi place parmi les maladies des peroxysomes.

L'oxalose est redoutable. Dans $30 \%$ des cas environ, la production excessive d'oxalate peut être ralentie par la pyridoxine ; dans

cliniques sont graves. La transplantation rénale se solde souvent par un échec : le dépôt d'oxalate de calcium se renouvelle et détruit le rein transplanté. Malgré la dialyse, l'oxalose extrarénale se développe. Watts et al. [3] ont réussi, après un premier échec, à effectuer une double transplantation du foie et du rein chez un malade de 23 ans, atteint d'oxalose primitive ; deux transplantations isolées du rein avaient antérieurement échoué chez ce malade. Grâce à la transplantation hépatique, une activité enzymatique a été restaurée ; la production d'oxalate a été freinée ; le rein transplanté a été protégé et sa fonction a été maintenue.

Cette observation montre comment la transplantation de certains organes peut corriger certains déficits enzymatiques; d'autres exemples sont connus, mettant à profit la transplantation de la moelle osseuse ou du foie. Quand le foie vient au secours du rein : bel exemple de solidarité.

J.P.-G

1. Danpure CJ, Jennings PR. Peroxisomal alanine : glyoxylate aminotransferase deficiency in primary hyperoxaluria type I. FEBS Lett $1986 ; 201: 20-4$.

2. Danpure CJ, Jennings PR, Watts RWE. Enzymological diagnosis of primary hyperoxaluria type I by measurement of hepatic alanine : glyoxylate aminotransferase activity. Lancet $1987 ; 1: 289-91$.

3. Watts RWE, Rolles $\mathrm{K}$, Morgan $\mathrm{SH}$, et al. Successful treatment of primary hyperoxaluria type I by combined hepatic and renal transplantation. Lancet $1987 ; 2$ : 474-5.

\section{BRÈVES}

$\mathrm{L} ' \alpha$ 2-antiplasmine ( $\alpha 2$ AP) fait partie de la famille des inhibiteurs des protéases à sérine, dont le mieux connu est $l^{\prime} \alpha 1$-antitrypsine $\left(\mathrm{m} / \mathrm{s} n^{\circ} 2\right.$, vol. 1 , p. 106 et $n^{\circ} 3$, vol. 3, p. 181). L'inhibition de l'enzyme résulte de la formation d'un complexe $1: 1$ stable entre le site réactif de l'inhibiteur et la sérine active de la protéase. L' $\alpha 2$ AP se lie à la plasmine par un site formé de I'Arg 364 et de la Met 365 (sur 452 acides aminés). Elle est présente dans le plasma normal à un taux voisin de $70 \mathrm{mg}$ par litre. On a décrit depuis 1978 des syndromes hémorragiques dus à un déficit en $\alpha 2$ AP. Récemment aux Pays-Bas ont été étudiés deux sujets déficients, un frère et une sœur ( $\alpha 2$ AP Enschede). L'activité fonctionnelle était de $3 \%$ de la normale sans baisse du taux de la protéine immunoréactive. Le séquençage de l'ADN génomique après clonage a montré une insertion de trois bases GCG, et donc la présence d'une alanine supplémentaire entre lès résidus 353 et 357, non loin du site actif. L'insertion d'un seul acide aminé suffit à modifier profondément les propriétés de l' $\alpha 2$ AP : elle garde son affinité pour la plasmine, mais, d'inhibiteur, elle est devenue substrat de cette protéase. Ainsi, à partir de cette observation inhabituelle, en modifiant expérimentalement la région voisine du site actif de l' $\alpha 2$ AP et des inhibiteurs apparentés, on peut désormais essayer de résoudre une question actuellement sans réponse: qu'est-ce qui, d'une antiprotéase, fait un inhibiteur, et non un substrat?

[Holmes W, et al. Science 1987 ; 238: 209-11.] 